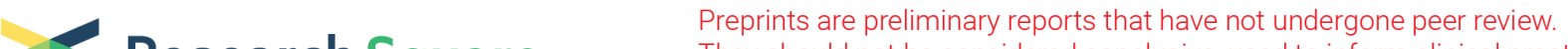 Research Square They should not be considered conclusive, used to inform clinical practice, or referenced by the media as validated information. \\ Lenvatinib Promotes the Antitumor Effects of Doxorubicin in Anaplastic Thyroid Cancer
}

\section{Xi Su}

Xi'an Jiaotong University Medical College First Affiliated Hospital

Jiaxin Liu

Xi'an Jiaotong University Medical College First Affiliated Hospital

\section{Haihong Zhang}

Xi'an Jiaotong University Medical College First Affiliated Hospital

\section{Qingqing Gu}

Xi'an Jiaotong University Medical College First Affiliated Hospital

\section{Xinrui Zhou}

Xi'an Jiaotong University Medical College First Affiliated Hospital

Meiju Ji (D miji0409@163.com)

Xi'an Jiaotong University Medical College First Affiliated Hospital

Demao Yao ( $\square$ ydm_723@163.com )

Xi'an Jiaotong University Medical College First Affiliated Hospital https://orcid.org/0000-0003-15676815

\section{Primary research}

Keywords: Anaplastic Thyroid Cancer, Lenvatinib, Doxorubicin, Combining therapy

Posted Date: May 20th, 2020

DOI: https://doi.org/10.21203/rs.3.rs-29005/v1

License: (c) (1) This work is licensed under a Creative Commons Attribution 4.0 International License. Read Full License

Version of Record: A version of this preprint was published at OncoTargets and Therapy on November 1st, 2020. See the published version at https://doi.org/10.2147/OTT.S278349. 


\section{Abstract \\ Background}

Anaplastic thyroid cancer (ATC) is a kind of rare thyroid cancer with very poor prognosis. It is one of the deadliest cancers in human due to the aggressive behavior and resistance to treatment. Doxorubicin has been approved in ATC treatment as a single agent, but monotherapy still shows no improvement of the total survival in advanced ATC. Lenvatinib was investigated with encouraging results in treating the patients with radioiodine-refractory differentiated thyroid cancer (DTC). However, antitumor efficacy of combination therapy with lenvatinib and doxorubicin remains largely unclear.

\section{Methods}

The antitumor efficacy of combination therapy with lenvatinib and doxorubicin on ATC cell proliferation and was assessed by the MTT assay and colony formation. Flow cytometry were employed to assess ATC cells' apoptosis and cell cycle arrest in response to combination therapy. Xenograft models were used to test its in vivo antitumor activity.

\section{Result}

Lenvatinib monotherapy was less effective than doxorubicin in treating ATC cell lines and xenografts model. The combination therapy of lenvatinib and doxorubicin significantly inhibited ATC cell proliferation and tumor growth in nude mice, and induced cell apoptosis and cell cycle arrest in compared to lenvatinib or doxorubicin monotherapy.

\section{Conclusion}

Lenvatinib promotes the antitumor effects of doxorubicin in ATC cell and xenografts model. Lenvatinib/doxorubicin combination may be a potential candidate therapeutic approach for ATC.

\section{Background}

ATC is the most aggressive type of thyroid cancer and one of the deadliest cancer in human [1, 2]. The incidence of ATC accounts for only less than $2 \%$ of thyroid cancer, but the mortality rate accounts for 15$40 \%$ of thyroid cancer $[3,4]$. ATC shows a rapid growth, easy metastasis and poor treatment outcomes, leading to a less than $10 \%$-year survival rate $[2,5]$. The standard treatment for ATC includes surgery, debulking, external beam radiation therapy, chemotherapy and multisystemic therapy $[6,7]$. Doxorubicin have been approved by American Thyroid Association (ATA) guidelines in ATC, however, monotherapy still shows no improvement of the total survival in advanced ATC [6]. Since combined therapy has shown 
better response than monotherapy $[8,9]$. Therefore, there is an urgent need to find new multiple targets combined with therapies against ATC.

Lenvatinib is a multi-target tyrosine kinase inhibitor (MKI) that targets fibroblast growth factor receptors 1-4 (FGFR1-4), vascular endothelial growth factor receptors 1-3 (VEGFR1-3), c-KIT and RET[10]. It has been approved by FDA and EMA as the second-line treatment for advanced radioiodine-refractory differentiated thyroid carcinoma (DTC) $[11,12]$. A recent study indicated lenvatinib could significantly reduce cell growth and increase apoptosis of ATC cells both in vitro and in vivo [13]. However, in a phase II study, postoperatively used lenvatinib to treat ATC patients indicated a response rate of only $17.4 \%$ [14]. Besides, another phase II study mainly assessing the safety and efficacy of lenvatinib in patient with thyroid cancer, have reported a manageable safety profile while a modest effectiveness of lenvatinib in ATC treatment [15]. These results indicated lenvatinib has a relative low efficacy when administered as a monotherapy to treat ATC.

Although it was verified in many studies that doxorubicin or lenvatinib as a monotherapy has limiting effectiveness, the combination of MKI with doxorubicin has shown increased anti-tumor activities in some cancers $[16,17]$. However, the antitumor activities of combining doxorubicin with lenvatinib in ATC were unknown. Thus, we performed a series of in vitro and in vivo experiments to explore the antitumor effectiveness of the combination of lenvatinib and doxorubicin in ATC.

\section{Methods}

\section{Cell culture and drugs administration}

ATC cell lines 8305C and 8505C were kindly provided by Dr. Haixia Guan (The First Affiliated Hospital of China Medical University, Shenyang, P. R. China). C643 were obtained from Dr. Lei Ye (Ruijin Hospital, Shanghai, P. R. China). All the cells were cultured in RPMI 1640 medium supplemented with $10 \%$ fetal bovine serum (Invitrogen Technologies, Inc., CA) at $37^{\circ} \mathrm{C}$. Cells were treated with lenvatinib (Selleck Chemicals, LLC) or/and doxorubicin (Selleck Chemicals, LLC) at the indicated concentrations and time points in some specific experiment. The lenvatinib and doxorubicin were dissolved in dimethylsulfoxide (DMSO), the same volume of DMSO was used as the vehicle control.

\section{Cell viability assay}

Cells (3000 to $4000 /$ well) were seeded in 96-well plates. After a 24 -h's culture, cells were treated with indicated doses of doxorubicin or/and lenvatinib for the indicated period time. Then $20 \mu \mathrm{L} \mathrm{of} 5 \mathrm{mg} / \mathrm{mL}$ 3(4,5-Dimethylthiazolyl-2)-2,5-diphenyltetrazolium bromide (MTT) was employed to assess the cell viability as described [18]. IC50 values were calculated with the Reed-Muench method [19].

\section{Colony formation assay}

Monolayer culture was performed to measure colony formation. Cells (3000 to 4000/well) were seeded into 12-well plate, and cultured with various doses of doxorubicin and lenvatinib, individually or in 
combination. The medium was refreshed every $72 \mathrm{~h}$. After 7-12 days of cultivation, colonies were fixed with $4 \%$ paraformaldehyde, and then washed with PBS and stained with a crystal violet solution. Each assay was carried out in triplicate.

\section{Cell apoptosis assay}

Cells were cultured with indicated doses of doxorubicin and lenvatinib, individually or in combination, for 48-72 h. Then cells were stained with Annexin V-FITC/PI Apoptosis Detection Kit (Roche Applied Science, Penzberg, Germany) according to the manufacturer's protocol. Apoptotic cells were measured by flow cytometer (BD Biosciences, $\mathrm{NJ}$ ). Each experiment was carried out in triplicate.

\section{Cell cycle analysis}

Cells in the exponential growth phase were serum starved for $12 \mathrm{~h}$. After individually or in combination co-culture with doxorubicin and lenvatinib for 48-72 h, cells were harvested, washed twice in PBS, and fixed in $70 \%$ ethanol on ice for $30 \mathrm{~min}$. Cells were then stained with PI solution $(50 \mu \mathrm{g} / \mathrm{mL} \mathrm{PI}, 50 \mu \mathrm{g} / \mathrm{mL}$ RNase A, 0.1\% Triton-X, 0.1 mM EDTA). Cell cycles were analyzed based on DNA content using a flow cytometer (BD Biosciences, $\mathrm{NJ}$ ).

\section{Animal studies}

8505C $\left(5 \times 10^{6}\right)$ were injected subcutaneously into the right armpit region of 5 - to 6-week-old female nude mice purchased from SLAC laboratory Animal Co., Ltd. (Shanghai, China) to establish xenograft mouse model. Mice were then randomly divided into four groups when tumor volume grew to $50-80 \mathrm{~mm}^{3}$. Doxorubicin ( $3 \mathrm{mg}$ per $\mathrm{kg}$ of body weight) was administered by intraperitoneal injection at a total volume of $0.2 \mathrm{~mL}$, and/or lenvatinib (5 mg per $\mathrm{kg}$ of body weight) was administered via oral route. The treatment was daily administered for 3 weeks. All mice were sacrificed, and tumors were collected and weighted $5 \mathrm{~h}$ after the final dose of drugs. Animal experiments were was approved by the Institutional Review Board of Xi'an Jiaotong University Health Science Center.

\section{Immunohistochemical (IHC) staining}

Tumor tissues were embedded in paraffin, sectioned at $4 \mu \mathrm{m}$, then cell proliferation ability was assessed by quantification of Ki-67 staining (percentage of positive cells). In brief, antihuman Ki-67 antibody (BD Pharmingen, CAT 550609) was 1:300 diluted, and immunostaining was done according to a standard protocol using DAB Substrate Kit (ZSGB-BIO). To evaluate the effect of different treatments on animal hepatocytes, we performed hematoxylin and eosin (H\&E) staining of liver, kidney and heart sections.

\section{Statistical analysis}

All the statistical analyses were performed with the SPSS statistical package (16.0, SPSS Inc. Chicago, IL). Unpaired student's t test was used to compare the means of two groups of data. One-way analysis of variance (ANOVA) followed by Bonferroni's multiple comparison test was used to compare differences 
between three or more groups. All values were expressed as the mean \pm standard deviation (SD). $P<0.05$ was considering statistically significant differences.

\section{Results}

\section{Lenvatinib potentiates proliferation-inhibitory effects of doxorubicin in ATC cells}

Doxorubicin have shown encouraging clinical activity in systematic therapy of ATC [6]. Thus, we first confirmed the proliferation inhibitory effects of doxorubicin in three ATC cell lines: 8305C, C643 and 8505C. As shown in Fig. 1a, doxorubicin significantly inhibited the proliferation of ATC cells in a dosedependent manner, with the IC50 values ranged from 5.84 to $13.31 \mathrm{nM}$. Since we have proved the antitumor activities of lenvatinib towards ATC cells [18], we next tested the effect of combination of lenvatinib and doxorubicin on the proliferation of ATC cells. As it showed in Fig. 1b, the combination of lenvatinib $(1 \mu \mathrm{M})$ and doxorubicin $(10 \mathrm{nM})$ indicated a synergistic inhibitory effect on proliferation in ATC cells as compared to either monotherapy.

The combination of lenvatinib and doxorubicin synergistically inhibits colony formation in thyroid cancer cells

Next, the growth-inhibitory effect of the combination of lenvatinib and doxorubicin was further explored with colony formation assay. As expected, lenvatinib as well as doxorubicin applied alone at the indicated concentrations showed the colony forming inhibitory effect in monolayer cultured ATC cell lines-8305C, C643 and 8505C (Fig. 2a-C).. Moreover, a combination of lenvatinib and doxorubicin caused a strongly enhanced inhibition of ATC cells' colony formation as compared to doxorubicin monotherapy (Fig. 2a-C). Collectively, these data suggested that the combination of doxorubicin with lenvatinib may largely enhance the tumor suppression function of doxorubicin.

\section{Lenvatinib enhances doxorubicin induced cell cycle arrest and apoptosis in ATC cells.}

Inhibition of cancer cell proliferation is usually connected with cell cycle arrest and cell apoptosis [20, 21]. Then we performed fluorescence-activated cell sorting analysis to investigate the contribution of lenvatinib and doxorubicin, individually or in combination, on ATC cell apoptosis and cell cycle arrest. Compared with the vehicle control, both lenvatinib and doxorubicin induced the apoptosis of ATC cells at the indicated concentrations (Fig. 3a-c). The addition of lenvatinib to doxorubicin synergistically increased the apoptotic cells in comparison to either lenvatinib or doxorubicin monotherapy (Fig. 3a-c). Besides, the addition of lenvatinib to doxorubicin induced more G2/M phase cell cycle arrest among three ATC cell lines compared with vehicle control or monotherapy (Fig. 4a-C).

Lenvatinib potentiates antitumor effects of doxorubicin in vivo. 
To determine whether the enhanced antitumor effects of doxorubicin by the addition of lenvatinib can also be observed in vivo, we established $8505 \mathrm{C}$ cell derived xenograft tumor in nude mice. These mice were treated with lenvatinib and doxorubicin, individually or in combination at the indicated concentrations and time points. As shown in Fig. 5a, the tumor weight of $8505 \mathrm{C}$ cell-derived xenograft tumors in the monotherapy groups were lighter than that in vehicle group; and the combination group was much lighter than that in monotherapy groups. Moreover, we assessed the cell proliferation in tumor tissues by IHC staining Ki-67. The number of Ki-67 positive cells in tumors from combination group was lower than that in monotherapy groups; while the monotherapy groups showed lower Ki-67 staining as compared to the vehicle control (Fig. 5b). Besides, histopathological evidences also showed that the combination therapy did not cause more significant organ's injury than that of monotherapy in mice (Fig. 5c). As a result, our data demonstrated the efficacy and safety of the combination of lenvatinib and doxorubicin for ATC treatment.

\section{Discussion}

ATC belongs to the undifferentiated thyroid cancer type and its incidence accounts for less than $2 \%$ among the thyroid cancer. However, the mortality rate of ATC remains high due to its easily metastasis and therapy resistance [5]. Surgical resection of the solid tumor, assisted with systemic adjuvant therapy, is the main therapy for ATC. However, the mainstay approaches were proved with limited effectiveness in some patients [22]. Indeed, with the development of molecular characterization exploring in ATC,

researchers have gain better understanding of its molecular pathogenesis in recent years [23]. These drive more novel treatments for anaplastic thyroid carcinoma [12]. Some of the new biological agents have showed potential in the ATC treatment. For example, ATC was found markedly expressed PD-L1 than DTC. Therefore, combining PD-L1 antibody with BRAF inhibitor PLX4720 or other kinase inhibitors could be used in ATC treatment $[24,25]$. In a phase I trial, three patients with ATC achieved complete remission and survived for more than 30 months with the administrated of combretastatin A-4 phosphate [26]. Another clinical study showed the complete response in a 49 year old women ATC patient by chemoradiation with dabrafenib [27]. These observations suggested that antitumor activity of the new potential agents against ATC were promising.

Nowadays, small molecule inhibitors targeting multiple tyrosine kinases have attracted great attention because of its clinical benefits in ATC treatment [12]. Therefore, some of them have been approved by the US FDA for management of different cancers including thyroid cancer such as vandetanib and lenvatinib, and some of them are in the processing of clinical trial [12]. The oral MKI lenvatinib was approved by the FDA in treating radioiodine refractory DTC, and some studies also indicated its modest antitumor effect in ATC patients. However, the using dose should be reduced owing to the grade 3 or higher adverse event $[12,14,15]$. These results suggested the lenvatinib monotherapy has limited clinical activity in ATC. Doxorubicin was approved by ATA guidelines as a conventional single-agent in ATC treatment [6], and some kind of MKIs was proved to enhance the anti-tumor effect of doxorubicin in different cancers [28, 29]. We thus investigated the feasibility of the combination of lenvatinib and doxorubicin for ATC treatment. 
Our data indicated that lenvatinib did show some effectiveness but less than that of doxorubicin in ATC cell lines and xenografts. Notably, we found that lenvatinib enhanced the effects of doxorubicin in ATC treatment both in vitro and in vivo. The combination of lenvatinib and doxorubicin synergistically inhibited ATC cells' proliferation and colony formation. Moreover, combining lenvatinib with potentiated doxorubicin induced apoptosis and cell cycle arrest compared with its monotherapy. Importantly, the dose of both lenvatinib and doxorubicin in combinatorial therapies didn't show additional side effects comparing to either monotherapy. These findings suggested a potential combination therapy in the management of ATC.

Actually, combining lenvatinib with conventional or newly developed anti-cancer drugs have been investigated as potential therapies $[8,30]$. Our data demonstrated that lenvatinib enhanced the antitumor effects of conventional anti-cancer drug doxorubicin in ATC not only in vitro but also in vivo. Mitogenactivated protein kinase (MAPK) pathway has been proved to play an important role in DNA repair in response to DNA damage [31,32]. We therefore supposed that the inhibition of receptor tyrosine kinases (RTKs) by lenvatinib impaired the ability of DNA repair. And combined with lenvatinib will enhanced the DNA damage caused by doxorubicin. However, further investigations into the detail molecular mechanisms are still needed. In summary, these preliminary results indicated that the lenvatinib/doxorubicin combination therapy may be an encouraging treatment for ATC.

\section{Conclusion}

Our data demonstrate that lenvatinib enhances the antitumor effects of doxorubicin in ATC. Lenvatinib/doxorubicin synergy was confirmed not only in vitro but also in vivo. These encouraging preliminary results suggest that lenvatinib/doxorubicin combination may be a potential therapeutic approach for ATC.

\section{Abbreviations}

DOX: doxorubicin; ATC: anaplastic thyroid cancer; RTKs: receptor tyrosine kinases

\section{Declarations}

\section{Consent for publication}

Not applicable

\section{Availability of data and materials}

All data generated or analysed during this study are included in this published article

\section{Acknowledgements}


We thank Dr. Haixia Guan (The First Affiliated Hospital of China Medical University, Shenyang, China) and Lei Ye (Ruijin Hospital, Shanghai, China) for kindly providing human thyroid cancer cell lines.

\section{Funding}

This work was supported by the Key Research and Development Projects of Shaanxi Province (No.2019SF-022).

\section{Authors' contributions}

XS and JXL performed all the experimental work. QQG and HHZ participated in data analysis. DMY and MJJ conceived and participated in the design of the study. The manuscript was written by XS, XRZ and DMY. All authors read and approved the final manuscript.

\section{Ethics Committee Approval and Patient Consent}

Animal experiments were was approved by the Institutional Review Board of Xi'an Jiaotong University Health Science Center.

\section{Competing interests}

The authors declare no competing interests.

\section{References}

1. Siegel RL, Miller KD, Jemal A. Cancer statistics, 2015. CA Cancer J Clin. 2015;65:5-29.

2. Are C, Shaha AR. Anaplastic thyroid carcinoma: biology, pathogenesis, prognostic factors, and treatment approaches. Ann Surg Oncol. 2006;13:453-64.

3. Siegel RL, Miller KD, Jemal A. Cancer statistics. 2019. Ca-a Cancer Journal for Clinicians. 2019;69:734.

4. Kitamura Y, Shimizu K, Nagahama M, Sugino K, Ozaki O, Mimura T, et al. Immediate causes of death in thyroid carcinoma: clinicopathological analysis of 161 fatal cases. J Clin Endocrinol Metab. 1999;84:4043-9.

5. Kebebew E, Greenspan FS, Clark OH, Woeber KA, McMillan A. Anaplastic thyroid carcinoma. Treatment outcome and prognostic factors. Cancer. 2005;103:1330-5.

6. Smallridge RC, Ain KB, Asa SL, Bible KC, Brierley JD, Burman KD, et al. American Thyroid Association guidelines for management of patients with anaplastic thyroid cancer. Thyroid. 2012;22:1104-39.

7. Ito K, Hanamura T, Murayama K, Okada T, Watanabe T, Harada M, et al. Multimodality therapeutic outcomes in anaplastic thyroid carcinoma: improved survival in subgroups of patients with localized primary tumors. Head Neck. 2012;34:230-7. 
8. Jing C, Gao Z, Wang R, Yang Z, Shi B, Hou P. Lenvatinib enhances the antitumor effects of paclitaxel in anaplastic thyroid cancer. Am J Cancer Res. 2017;7:903-12.

9. Yau T, Lo CY, Epstein RJ, Lam AK, Wan KY, Lang BH. Treatment outcomes in anaplastic thyroid carcinoma: survival improvement in young patients with localized disease treated by combination of surgery and radiotherapy. Ann Surg Oncol. 2008;15:2500-5.

10. Cabanillas ME, Habra MA. Lenvatinib: Role in thyroid cancer and other solid tumors. Cancer Treat Rev. 2016;42:47-55.

11. Schlumberger M, Jarzab B, Cabanillas ME, Robinson B, Pacini F, Ball DW, et al. A Phase II Trial of the Multitargeted Tyrosine Kinase Inhibitor Lenvatinib (E7080) in Advanced Medullary Thyroid Cancer. Clin Cancer Res. 2016;22:44-53.

12. Ferrari SM, Elia G, Ragusa F, Ruffilli I, La Motta C, Paparo SR, et al. Novel treatments for anaplastic thyroid carcinoma. Gland Surg. 2020;9:28-42.

13. Ferrari SM, Bocci G, Di Desidero T, Elia G, Ruffilli I, Ragusa F, et al. Lenvatinib exhibits antineoplastic activity in anaplastic thyroid cancer in vitro and in vivo. Oncol Rep. 2018;39:2225-34.

14. Iwasaki $H$, Yamazaki $H$, Takasaki H, Suganuma N, Nakayama $H$, Toda $S$, et al. Lenvatinib as a novel treatment for anaplastic thyroid cancer: A retrospective study. Oncol Lett. 2018;16:7271-7.

15. Takahashi S, Kiyota N, Yamazaki T, Chayahara N, Nakano K, Inagaki L, et al. A Phase II study of the safety and efficacy of lenvatinib in patients with advanced thyroid cancer. Future Oncol. 2019;15:717-26.

16. Estupinan O, Santos L, Rodriguez A, Fernandez-Nevado L, Costales P, Perez-Escuredo J, et al. The multikinase inhibitor EC-70124 synergistically increased the antitumor activity of doxorubicin in sarcomas. Int J Cancer. 2019;145:254-66.

17. Badinloo M, Esmaeili-Mahani S. Phosphatidylinositol 3-kinases inhibitor LY294002 potentiates the cytotoxic effects of doxorubicin, vincristine, and etoposide in a panel of cancer cell lines. Fundam Clin Pharmacol. 2014;28:414-22.

18. Su X, Shen Z, Yang Q, Sui F, Pu J, Ma JJ, et al. Vitamin C kills thyroid cancer cells through ROSdependent inhibition of MAPK/ERK and PI3K/AKT pathways via distinct mechanisms. Theranostics. 2019;9:4461-73.

19. Welkos S, O'Brien A. Determination of median lethal and infectious doses in animal model systems. Methods Enzymol. 1994;235:29-39.

20. Hanahan D, Weinberg RA. Hallmarks of cancer: the next generation. Cell. 2011;144:646-74.

21. Hanahan D, Weinberg RA. The hallmarks of cancer. Cell. 2000;100:57-70.

22. Keutgen XM, Sadowski SM, Kebebew E. Management of anaplastic thyroid cancer. Gland Surg. 2015;4:44-51.

23. Landa I, Ibrahimpasic T, Boucai L, Sinha R, Knauf JA, Shah RH, et al. Genomic and transcriptomic hallmarks of poorly differentiated and anaplastic thyroid cancers. J Clin Invest. 2016;126:1052-66. 
24. Iyer PC, Dadu R, Gule-Monroe M, Busaidy NL, Ferrarotto R, Habra MA, et al. Salvage pembrolizumab added to kinase inhibitor therapy for the treatment of anaplastic thyroid carcinoma. $\mathrm{J}$ Immunother Cancer. 2018;6:68.

25. Brauner E, Gunda V, Vanden Borre P, Zurakowski D, Kim YS, Dennett KV, et al. Combining BRAF inhibitor and anti PD-L1 antibody dramatically improves tumor regression and anti tumor immunity in an immunocompetent murine model of anaplastic thyroid cancer. Oncotarget. 2016;7:17194-211.

26. Dowlati A, Robertson K, Cooney M, Petros WP, Stratford M, Jesberger J, et al. A phase I pharmacokinetic and translational study of the novel vascular targeting agent combretastatin a-4 phosphate on a single-dose intravenous schedule in patients with advanced cancer. Cancer Res. 2002;62:3408-16.

27. Lim AM, Taylor GR, Fellowes A, Cameron L, Lee B, Hicks RJ, et al. BRAF Inhibition in BRAFV600EPositive Anaplastic Thyroid Carcinoma. J Natl Compr Canc Netw. 2016;14:249-54.

28. Aggerholm-Pedersen N, Demuth C, Safwat A, Meldgaard P, Kassem M, Sandahl Sorensen B. Dasatinib and Doxorubicin Treatment of Sarcoma Initiating Cells: A Possible New Treatment Strategy. Stem Cells Int. 2016;2016:9601493.

29. Chen W, Liu I, Tomiyasu H, Lee J, Cheng C, Liao AT, et al. Imatinib enhances the anti-tumour effect of doxorubicin in canine B-cell lymphoma cell line. Vet J. 2019;254:105398.

30. Chen WX, Li GX, Hu ZN, Zhu P, Zhang BX, Ding ZY. Significant response to anti-PD-1 based immunotherapy plus lenvatinib for recurrent intrahepatic cholangiocarcinoma with bone metastasis: A case report and literature review. Medicine. 2019;98:e17832.

31. Wood CD, Thornton TM, Sabio G, Davis RA, Rincon M. Nuclear localization of p38 MAPK in response to DNA damage. Int J Biol Sci. 2009;5:428-37.

32. Ulm R, Revenkova E, di Sansebastiano GP, Bechtold N, Paszkowski J. Mitogen-activated protein kinase phosphatase is required for genotoxic stress relief in Arabidopsis. Genes Dev. 2001;15:699709.

\section{Figures}



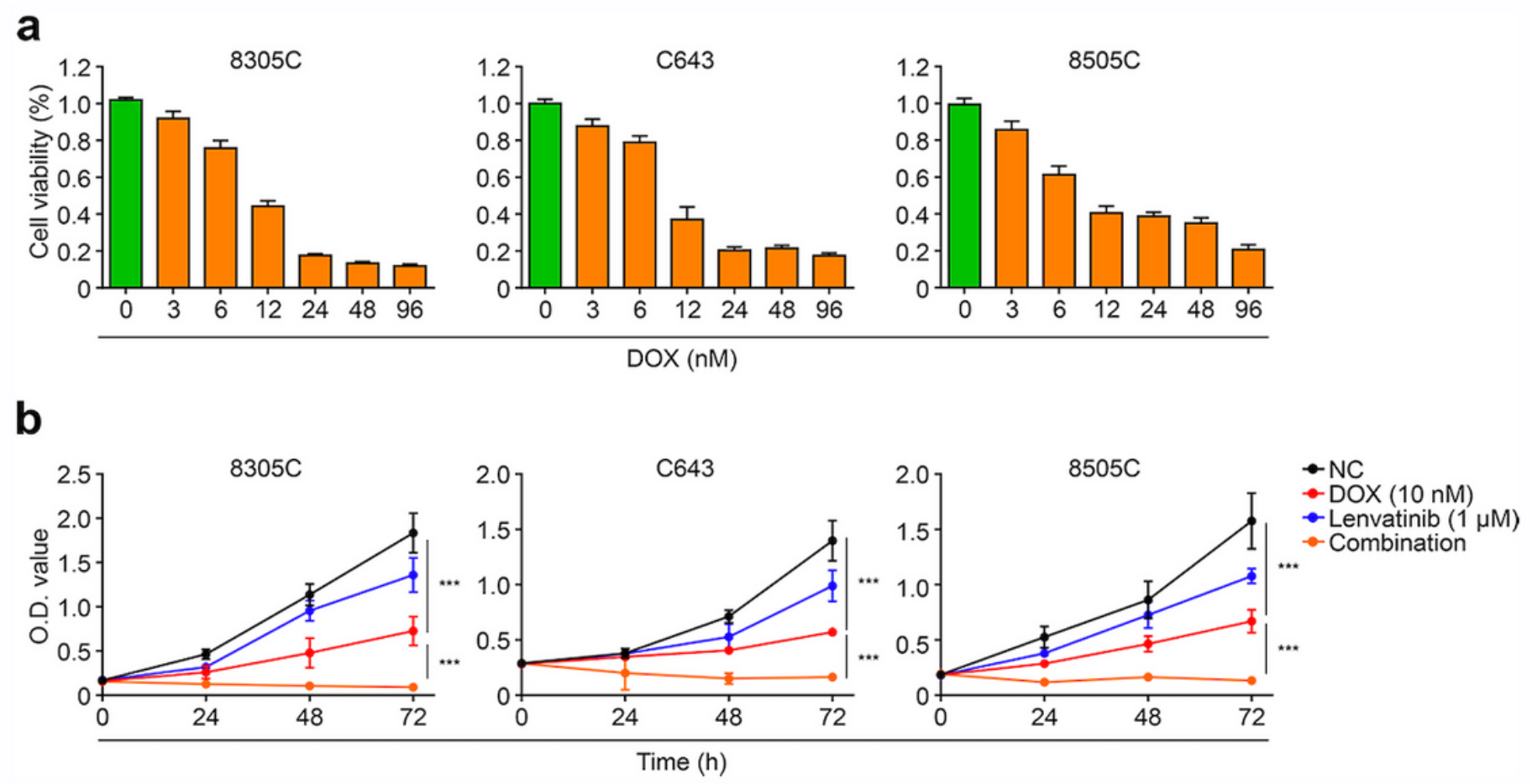

\section{Figure 1}

The combination of lenvatinib and doxorubicin inhibited the proliferation of different ATC cells. a Cells were treated with different concentrations of doxorubicin for $48 \mathrm{~h}$. MTT assay was employed to evaluate cell viability. $b$ MTT assay was used to evaluate the proliferation inhibitory effect of combining therapy. Data were presented as mean $\pm S D * \star \star, P<0.001$. 
a
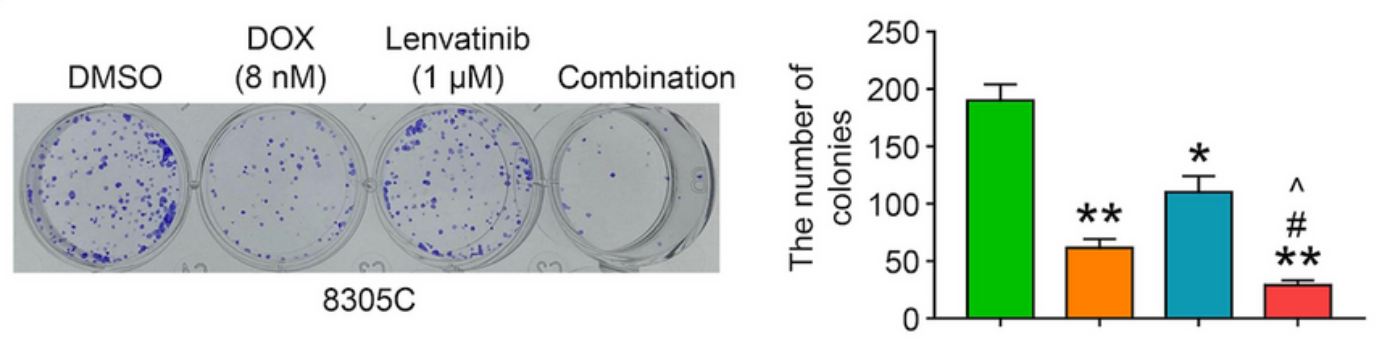

b
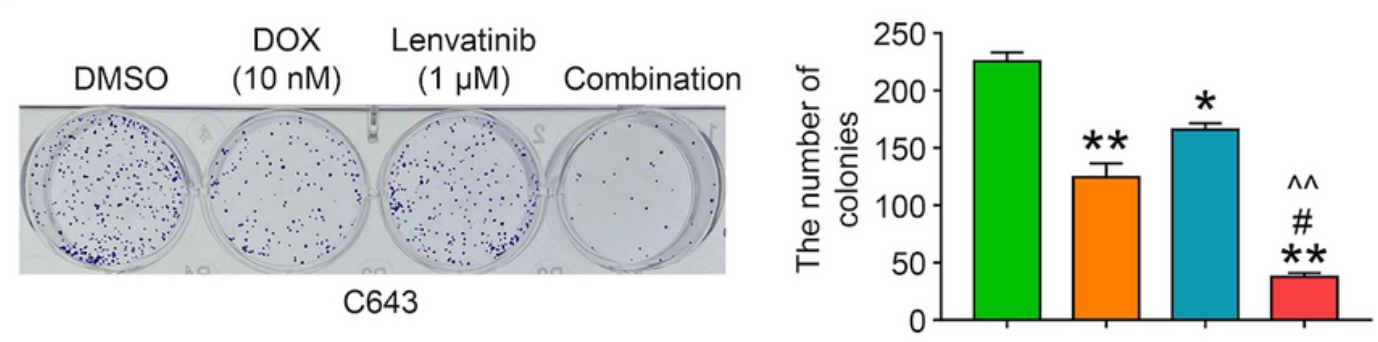

DMSO

DOX (8 nM)

Lenvatinib $(1 \mu \mathrm{M})$

Combination

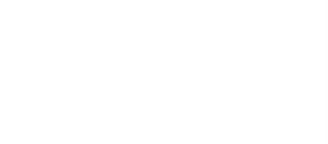

C
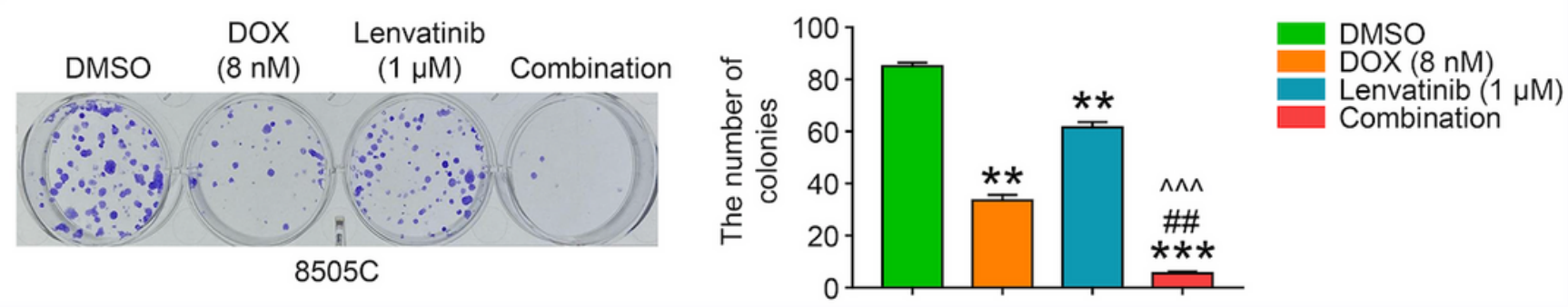

\section{Figure 2}

The combination of lenvatinib and doxorubicin inhibited the colony formation of different ATC cells. Representative figures of colony formation in $8305 \mathrm{C}$ a, C643 b and $8505 \mathrm{C} c$ cells were shown in the left panel. The number of colonies was calculated and presented in right panel. Data were presented as mean \pm SD. *, $\mathrm{P}<0.05$; $* \star, \mathrm{P}<0.01$; $* \star \star, \mathrm{P}<0.001$. *, compared with DMSO group; \#, compared with DOX group; $\wedge$, compared with Lenvatinib group. 
a

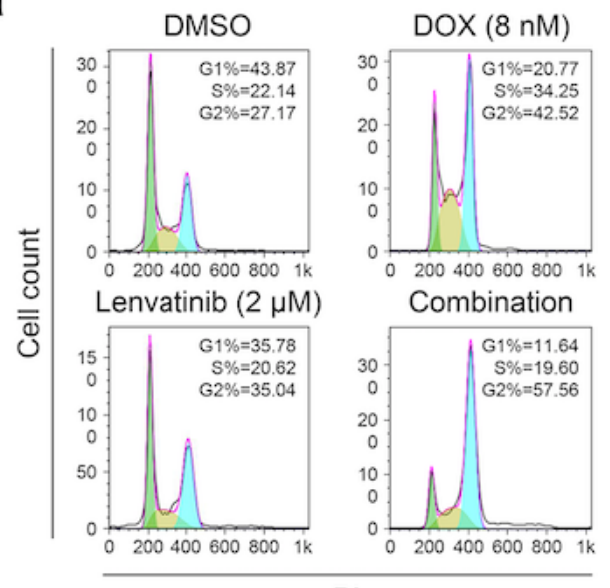

b

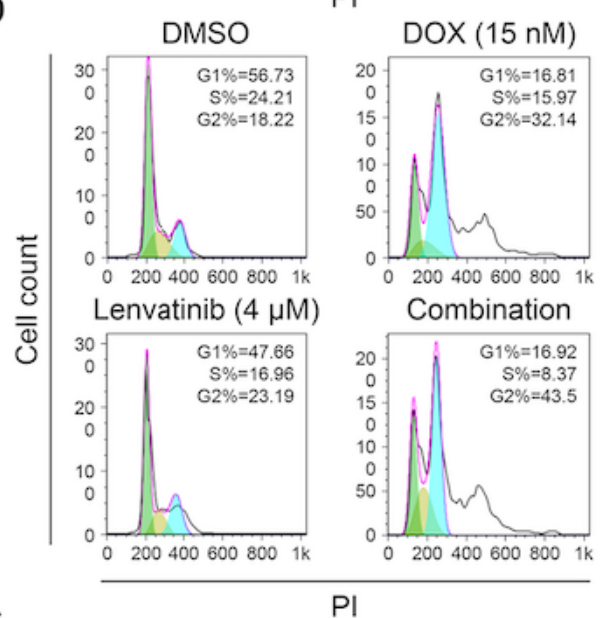

C

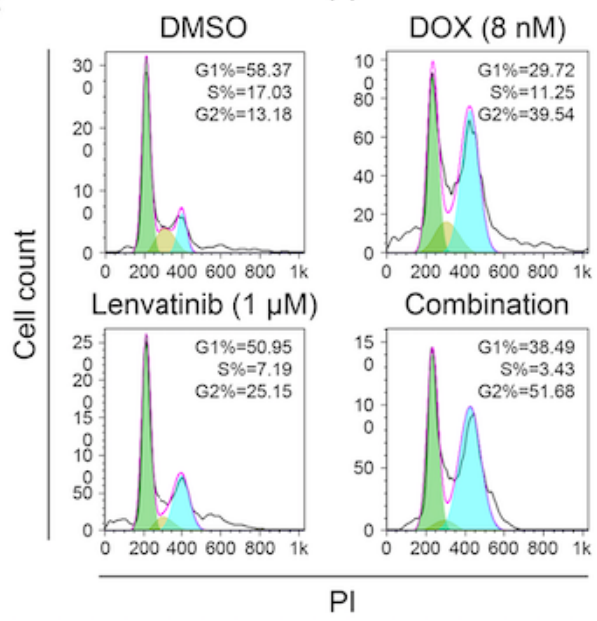

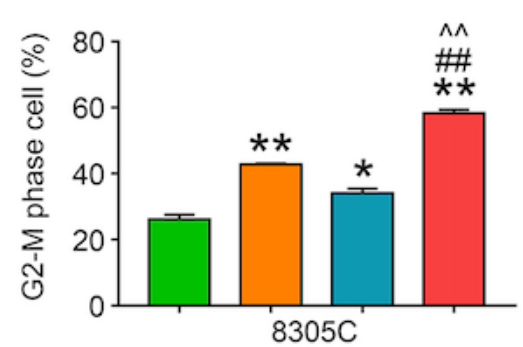
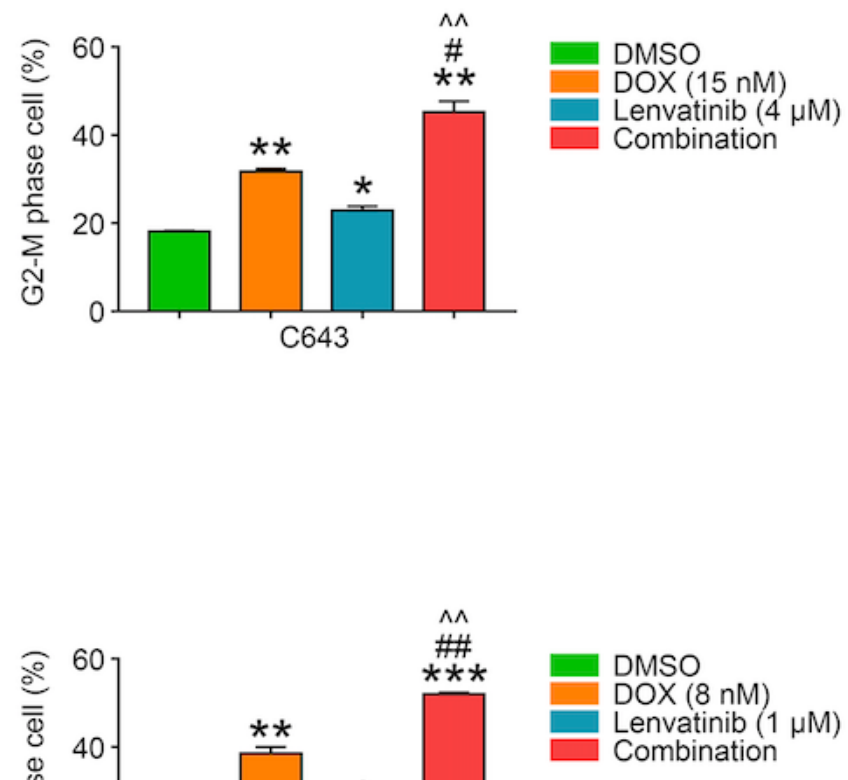

\section{Figure 3}

The combination of lenvatinib and doxorubicin synergistic induced apoptosis of ATC cells. 8305C a, C643 b and 8505C c were treated with lenvatinib or/and doxorubicin with the indicated concentration for 48-72 h. Apoptosis was measured by flow cytometry. Representative figures showed the percentage of apoptotic cells (left panel). Quantitative illustration of apoptotic cells was shown in right panel. Data were 
presented as mean \pm SD. *, $\mathrm{P}<0.05$; **, $\mathrm{P}<0.01$; ***, $\mathrm{P}<0.001$. *, compared with DMSO group; \#, compared with DOX group; ${ }^{\wedge}$, compared with Lenvatinib group.

a
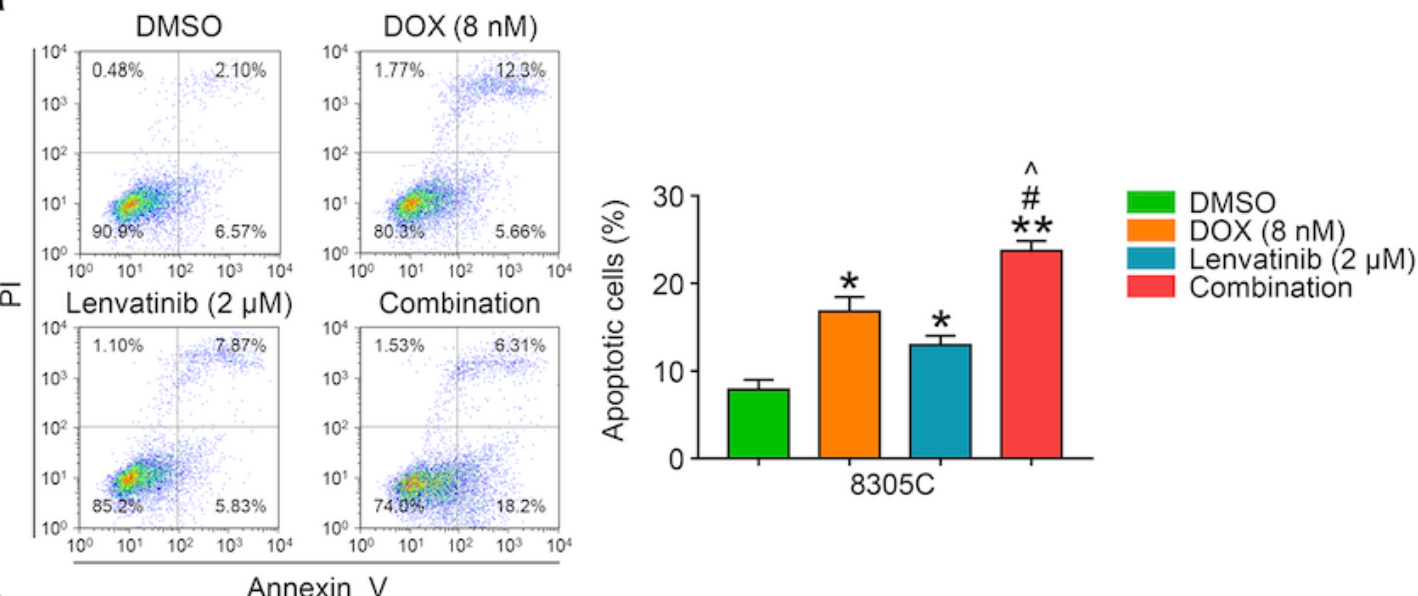

b
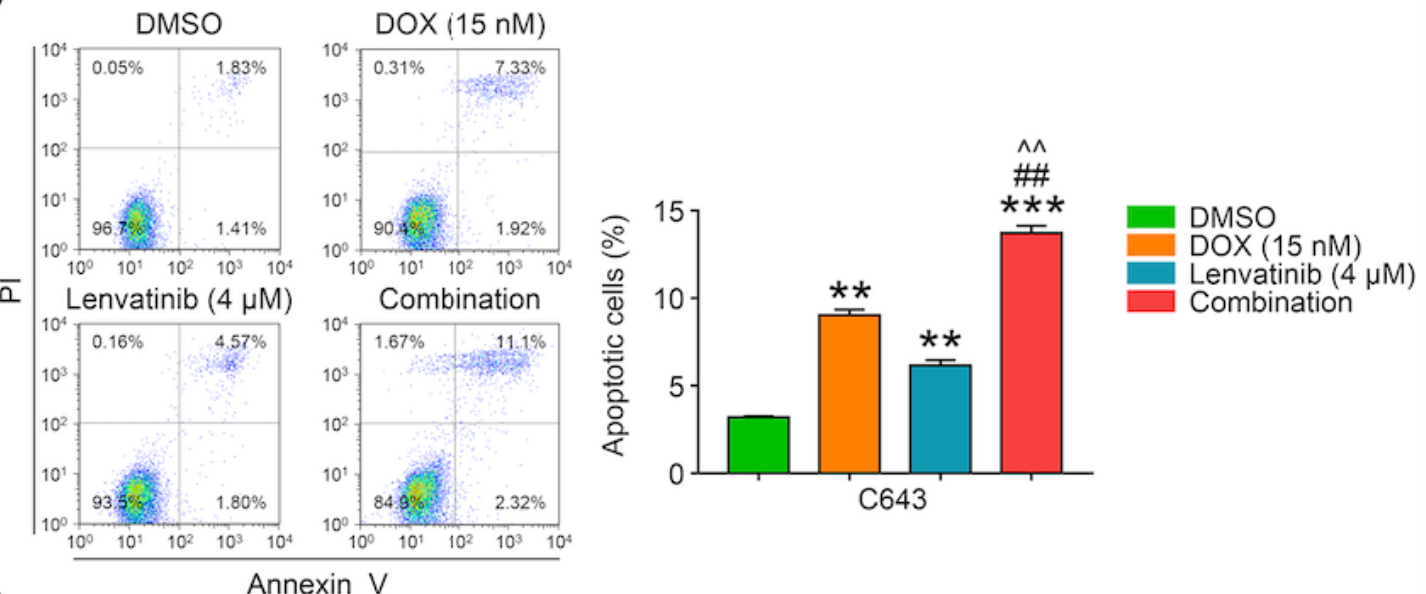

C
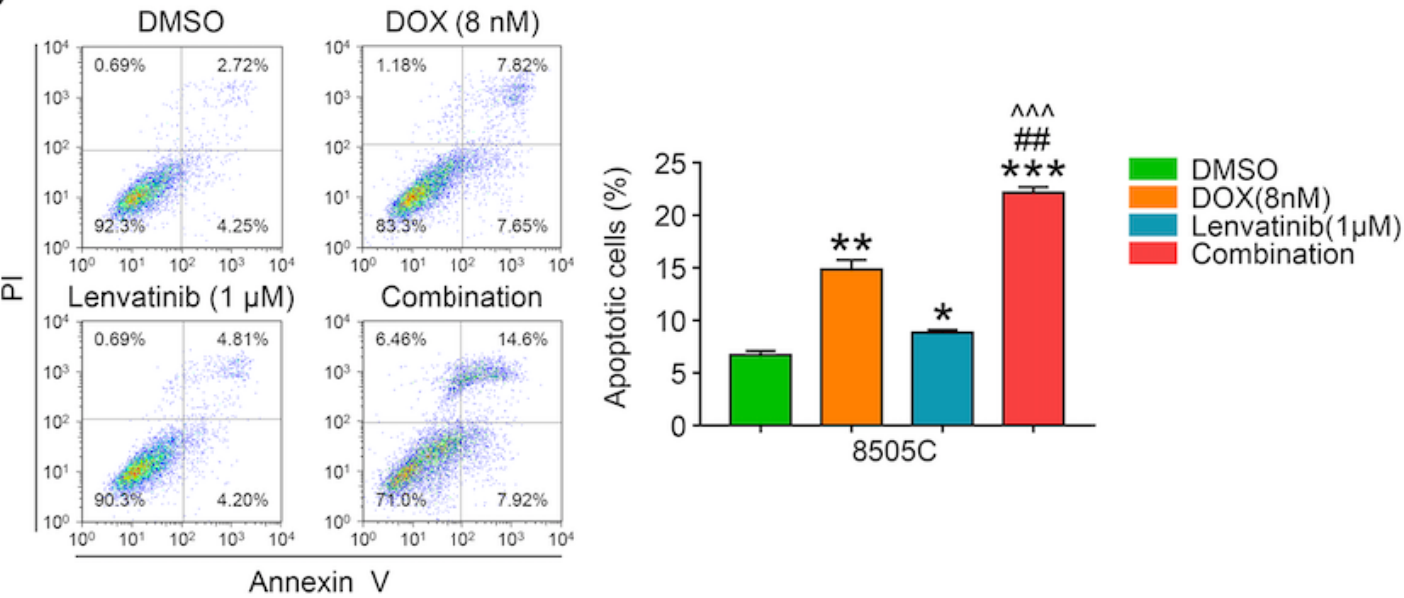

Figure 4

The combination of lenvatinib and doxorubicin synergistic induced G2/M phase cell cycle arrest ATC cells. 8305C a, C643 b and 8505C c were treated with lenvatinib or/and doxorubicin with the indicated concentration for 48-72 h. DNA content was measured by flow cytometry. Representative figures showed 
the flow cytometric histograms (left panel). Quantitative analysis of G2/M phase cells was shown in right panel. Data were presented as mean $\pm \mathrm{SD} .{ }^{*}, \mathrm{P}<0.05 ;{ }^{*}, \mathrm{P}<0.01$; ${ }^{* \star}, \mathrm{P}<0.001$. ${ }^{*}$, compared with DMSO group; \#, compared with DOX group; ${ }^{\wedge}$, compared with Lenvatinib group.

\section{a}

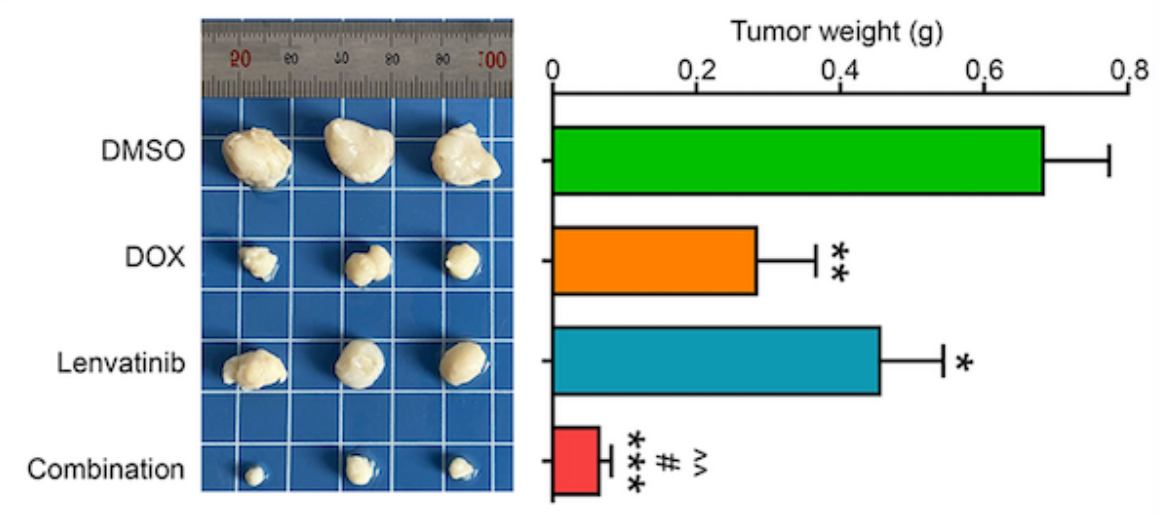

b
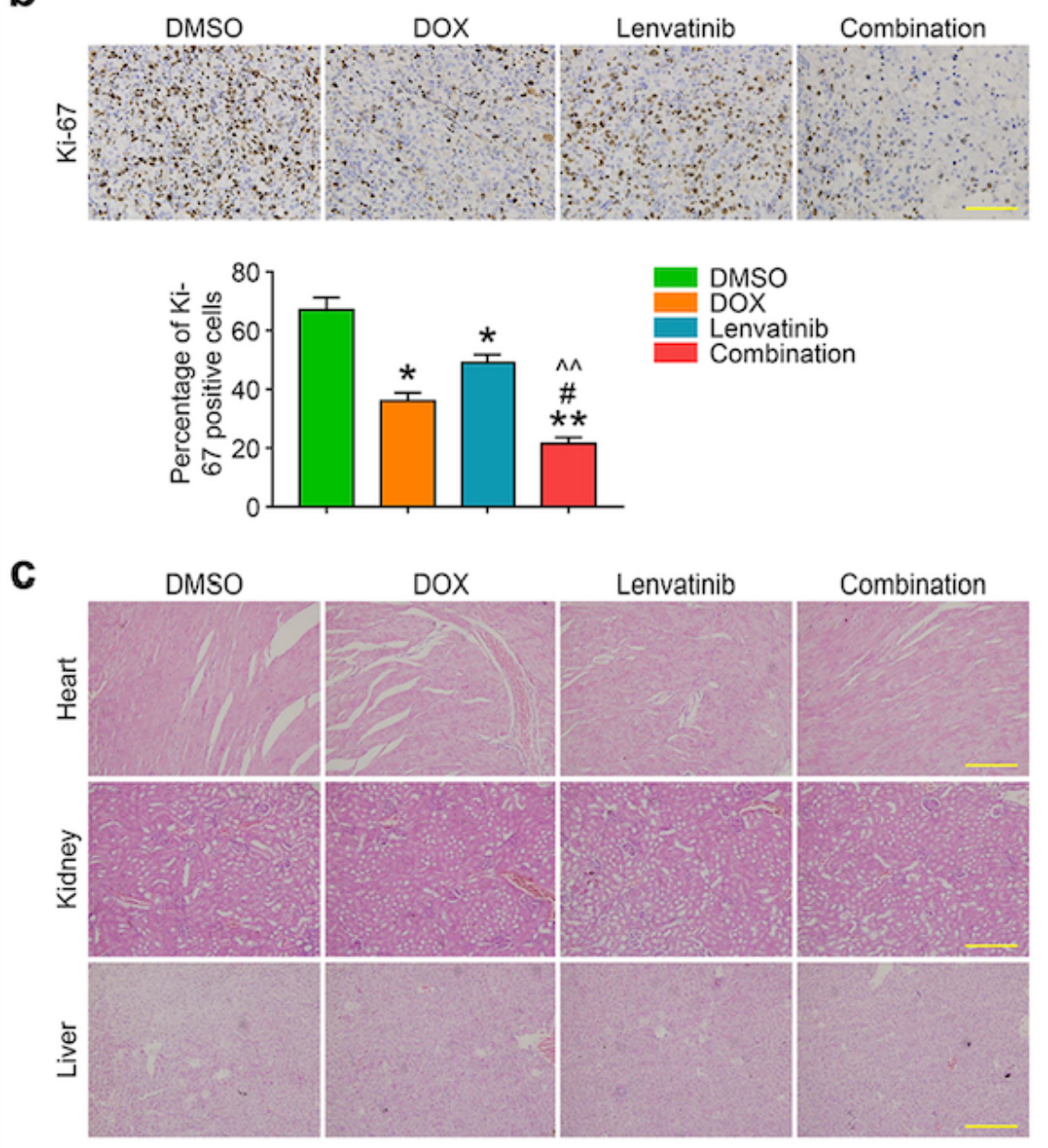

Figure 5

Validation of combining lenvatinib and doxorubicin as a functional therapy in vivo. a Representative images showed the isolated tumors from mice in different groups (left panel), and the bar chart 
illustrated the tumor weight. $\mathrm{b}$ The levels of $\mathrm{Ki}-67$ proteins in the indicated xenograft tumors were measured by IHC assay (upper panels). Statistical analysis of the percentage of Ki-67 positive cells was shown in lower panels. Scale bars, $200 \mu \mathrm{m}$. c Representative H\&E stained heart, kidney and liver sections from the indicated mice. Scale bars, $200 \mu \mathrm{m}$. Data were presented as mean $\pm \mathrm{SD}$. ${ }^{*}, \mathrm{P}<0.05 ; * \star, P<0.01$; $\star \star \star, P<0.001 .{ }^{*}$, compared with DMSO group; \#, compared with DOX group; ${ }^{\wedge}$, compared with Lenvatinib group. 